\title{
Gemella morbillorum septicemia with hepatorenal encephalopathy - An unusual fatal case
}

\author{
Authors \\ Dr Mayuri Shrivastava, Dr Santwana Verma, Dr Digvijay Singh, Dr Vineeta Sharma, \\ Dr Anumeha Gupta \\ Dept of Microbiology, Indira Gandhi Medical Collge, Shimla \\ Corresponding Author \\ Dr Mayuri Shrivastava \\ Dept of Microbiology, Indira Gandhi Medical Collge, Shimla, India
}

\section{Introduction}

Gemella morbillorum was earlier known as Streptococcus morbillorum. The present classification (1988) was based on elucidation of significant differences on DNA sequencing ${ }^{[1]}$. It is a facultative anaerobe and is part of commensal flora of human oropharyngeal, upper respiratory and gastrointestinal tracts ${ }^{[1]}$. These Gram-positive cocci were first isolated from humans in 1917 and are reported as cause of endocarditis, bacteremia, sepsis, endo-vascular abscesses and colon cancer $^{[1,2]}$. Infections due to G.morbillorum are frequently reported in children or elderly patients with poor immune status, or other predisposing factors like congenital heart disease, previous dental procedure, poor dental hygiene or intravenous drug abuse ${ }^{[3-6]}$. We aim to generate awareness about the pathogenic potential of this bacterium as misidentification as viridians Streptococci may lead to fatal consequences.

\section{Case Report}

A 70-year-old male was brought to the emergency department of the institute with three day history of fever associated with chills and sweating. He had altered sensorium and difficulty in breathing. On general physical examination patient was febrile. Pallor and cyanosis were present but there was no icterus or clubbing of nails. There was bilateral pitting pedal edema. Blood pressure (BP) was not recordable and oxygen saturation was low at the time of presentation. Initial management in the form of intravenous fluids, inotropic agents and oxygen inhalation were initiated for maintenance of blood pressure and oxygen saturation. Subsequently, BP was recorded, diastole as $70 \mathrm{mmHg}$, systole as $100 \mathrm{~mm} \mathrm{Hg}$ and pulse $120 / \mathrm{min}$.

The patient was a known case of hypertension and revealed history of taking antihypertensive medication irregularly. There was history of dyspnoea on exertion since two years while walking uphill. The personal history reveals that the patient consumed alcohol and was also a smoker.

On systemic examination of central nervous system patient was unconscious with Glasgow Coma Scale of E1V2M2 and plantar reflexes were 
up going. Examination of respiratory system revealed crackles all over the chest bilaterally up to interscapular area. Features of cardiomyopathy were observed on examination of cardiovascular system and inspection of other systems was unremarkable.

The hematological and biochemical investigations showed haemoglobin $11.9 \mathrm{gm} / \mathrm{dl}$, total leucocyte count $20.3 \times 10^{3} / \mu 1$ and platelet count $1,83,000 / \mu 1$. Random blood sugar was $110 \mathrm{mg} / \mathrm{dl}$, kidney function test revealed total protein $4.9 \mathrm{~g} / \mathrm{dl}$, albumin $2.5 \mathrm{~g} / \mathrm{dl}$, globulin $2.4 \mathrm{~g} / \mathrm{dl}$, blood urea nitrogen $55 \mathrm{mg} / \mathrm{dl}$, creatinine $2.9 \mathrm{mg} / \mathrm{dl}$ and uric acid $4.2 \mathrm{mg} / \mathrm{dl}$. There was electrolyte imbalance with sodium level $133 \mathrm{mmol} / \mathrm{L}$, potassium $36.4 \mathrm{~mm} / \mathrm{l}$ and chloride $80 \mathrm{mmol} / \mathrm{l}$. Lipid profile revealed cholesterol $106 \mathrm{mg} / \mathrm{dl}$, triglycerides $82 \mathrm{mg} / \mathrm{dl}, \mathrm{HDL} 83 \mathrm{mg} / \mathrm{dl}$ and LDL $34 \mathrm{mg} / \mathrm{dl}$. In the liver function tests, conjugated bilirubin was 0.68g/l, total bilirubin 1.25g/l, ALT $1349 \mathrm{U} / \mathrm{L}$, AST $1192 \mathrm{U} / \mathrm{L}$ and alkaline phosphatase was $115 \mathrm{U} / \mathrm{L}$. Magnetic resonance imaging of brain demonstrated normal parenchyma with differentiation of grey and white matter. T2 flair revealed periventricular hyper intense with microangiopathic changes.

In the laboratory workup for fever, blood culture was performed in automated BD BACTEC ${ }^{\text {TM }}$ FX blood culture system which indicated positive results. Subcultures were done on blood agar and Mc Conkey's agar and incubated at $37^{\circ} \mathrm{C}$ for 24 hours. Blood agar plate grew pin point, circular, convex, translucent, shiny, smooth $\alpha$-hemolytic colonies which demonstrated gram positive cocci on stained smears. Final identification was established as Gemella morbillorum on BD automated IDST Phoenix System with confidence limits of $98 \%$. IgM ELISA for scrub typhus was negative and culture of urine sample was sterile.

Empirical therapy in the form of piperacillintazobactum $4.5 \mathrm{gm}$ IV twice a day and paranteral levofloxacin 750gm daily was started. Antibiotic susceptibility was performed by Kirby Baeur's disc diffusion test based on which meropenem $500 \mathrm{mg}$, linezolid 600mg and doxycycline 100mg intravenously twice a day were initiated. The patient showed some improvement at first but died after few days due to multiorgan failure.

\section{Discussion}

Gemella morbillorum is a commensel and an unusual cause of human infections. As opportunistic pathogen, it causes serious and fatal infections in both pediatric and adult population with immunocompromised conditions including steroid therapy, diabetes mellitus and infection with human immunodeficiency virus ${ }^{[5-8]}$. Other predisposing risk factors are underlying heart disease, poor dental hygiene, intravenous drug use, old age, dental and gastrointestinal procedures $^{[1,4,6]}$. The present case was an elderly male with poor dental hygiene. Periodontitis is reported as the most frequent source of Gemella morbillorum $^{[3]}$. It is implicated as a cause of endocarditis and sepsis in some cases ${ }^{[1-5]}$. Involvement of heart valves primarily the aortic valves has been described by some authors. ${ }^{[6,9]}$ The present case though not diagnosed with endocarditis yet had cardiomyopathy.

Majority of cases reported earlier show favourable respone to therapy with penicillins, cephalosporins, clindamycin and vancomycin together with management of the primary disease ${ }^{[1-3]}$. Recommended treatment is 4 to 6 weeks of antibiotic therapy with penicillin based regimen but lately emergence of resistance to penicillin has been documented in which case vancomycin is an effective alternative $\operatorname{drug}^{[1,10]}$. In the present case, despite initial response to antimicrobial treatment, the co-morbid conditions of severe renal injury, probably due to uncontrolled hypertension, and hepatic derangement leading to encephalopathy may have been responsible for the fatal outcome.

\section{Conclusion}

Gemella morbillorum is a less known pathogen causing infections in high risk individuals. It should be considered when standard methods identify slow growing catalase-negative grampositive cocci. These organisms are generally 
reported as contaminants as they appear similar to $\alpha$ - hemolytic Streptococci which are usually commensals and clinicians and laboratory physicians are unfamiliar with the pathogenic potential of Gemella morbillorum. Patients often go untreated which may lead to fatal outcome. Identification and prompt treatment of infection due to Gemella morbillorum and other underlying disease may be life saving.

\section{References}

1. Military James EH. Multisystem organ failure due to Gemella morbillorum native valve endocarditis. Military Medicine. 2010;175:923-925.

2. Lokesh Shahani L. Gemella morbillorum prosthetic aortic valve endocarditis BMJ Case Rep. 2014.doi:10.1136/bcr-2014207304.

3. Andrew Czarnecki, George HFO, Pueroni P, Trepman E, Embil F. Am J O. Gemella morbillorum septic arthritis of the knee and infective endocarditis. Am J Ortho. 2007; 36(1):E7-E9.

4. Stroup JS, Bransteitter A, Reust R. Infective endocarditis caused by Gemella species. Infectious Diseases in Clinical Practice. 2007;15(3):203-205.

5. Purcell LK, Finley JP, Chen R, Lovgren M, Halperin SA. Gemella species endocarditis in a child. Canadian Journal of Infectious Diseases. 2001;12(5):317320.

6. Akiyama K, Taniyasu N, Hirota J, Iba Y, Maisawa K. Recurrent aortic valve endocarditis caused by Gemella morbillorum: report of a case and review of the literature. Japanese Circulation Journal. 2001; 65(11):997-1000.

7. Zakir RM, Dehneh A, Dabu L, Kapila R, Saric. Mitral biprosthetic valve endocarditis caused by an unusual microorganism, Gemella morbillorum in an intravenous drug user. Journal of Clinical Microbiology. 2004; 42(10):48934896.

8. Gervasoni C, Ridolfo A, Antinori S, Bini $\mathrm{T}$, Esposito R. Fatal septic shock due to Gemella morbillorum in two HIV-positive patients. Clin Microbiol Infect. 1996;2:6566.

9. Taimur S, Madiha R, Samar F, Bushra J. Gemella morbillorum endocarditis in a patient with a bicuspid aortic valve. Hellenic Journal of Cardiology. 2010;51(2):183-186.

10. Woo PC, Lau SK, Fung AM, et al. Gemella bacteremia characterized by $16 \mathrm{~S}$ ribosomal RNA gene sequencing. J Clin Pathol 2003;56:690-693. 